\title{
Formation of Néel-type skyrmions in an antidot lattice with perpendicular magnetic anisotropy
}

\author{
S. Saha $\odot,{ }^{1,2, *}$ M. Zelent $\odot,{ }^{3, \dagger}$ S. Finizio $\odot,{ }^{2}$ M. Mruczkiewicz, ${ }^{4}$ S. Tacchi $\odot,{ }^{5}$ A. K. Suszka, ${ }^{1,2}$ S. Wintz, ${ }^{2,6}$ N. S. Bingham, ${ }^{1,2,7}$ \\ J. Raabe, ${ }^{2}$ M. Krawczyk, ${ }^{4}$ and L. J. Heyderman (1) ${ }^{1,2}$ \\ ${ }^{1}$ Laboratory for Mesoscopic Systems, Department of Materials, ETH Zurich, 8093 Zurich, Switzerland \\ ${ }^{2}$ Paul Scherrer Institut, 5232 Villigen PSI, Switzerland \\ ${ }^{3}$ Faculty of Physics, Adam Mickiewicz University in Poznan, Umultowska 85, Poznan PL-61-614, Poland \\ ${ }^{4}$ Institute of Electrical Engineering, Slovak Academy of Sciences, Dubravska Cesta 9, SK-841-04 Bratislava, Slovakia \\ ${ }^{5}$ Istituto Officina dei Materiali del CNR (CNR-IOM), Sede Secondaria di Perugia, c/o Dipartimento di Fisica e Geologia, \\ Università di Perugia, I-06123 Perugia, Italy \\ ${ }^{6}$ Helmholtz-Zentrum Dresden-Rossendorf, 01328 Dresden, Germany \\ ${ }^{7}$ Department of Applied Physics, Yale University, New Haven, Connecticut 06511, USA
}

(Received 27 May 2019; revised manuscript received 30 September 2019; published 25 October 2019)

\begin{abstract}
Magnetic skyrmions are particlelike chiral spin textures found in magnetic films with out-of-plane anisotropy and are considered to be potential candidates as information carriers in next generation data storage devices. Despite intense research into the nature of skyrmions and their dynamic properties, there are several key challenges that still need to be addressed. In particular, the outstanding issues are the reproducible generation, stabilization, and confinement of skyrmions at room temperature. Here, we present a method for the capture of magnetic skyrmions in an array of defects in the form of an antidot lattice. We find that inhomogeneity in the total effective field produced by the antidot lattice is important for the formation of skyrmions which are mainly stabilized by the dipolar interaction. With micromagnetic simulations and scanning transmission x-ray microscopy we elucidate that the formation of skyrmions within the antidot lattice depends on the lattice constant and that, below a certain lattice constant, the skyrmion formation is suppressed. Based on our results we propose that, by varying the lattice constant, we can modify the probability of skyrmion formation in different parts of a sample by specific patterning. This provides another platform for experimental investigations of skyrmions and skyrmion-based devices.
\end{abstract}

DOI: 10.1103/PhysRevB.100.144435

\section{INTRODUCTION}

In materials exhibiting perpendicular magnetic anisotropy (PMA), noncollinear spin textures can be stabilized in weak magnetic fields or even at remanence. In particular, labyrinth domains can shrink to form isolated magnetic skyrmions $[1,2]$, which are topologically nontrivial stable spin textures characterized by a nonzero winding number with topological charge [3]. The antisymmetric exchange interaction, known as the Dzyaloshinskii-Moriya interaction (DMI) [4], which can arise due to large spin-orbit coupling in the presence of broken inversion symmetry in the material, helps to stabilize the chiral magnetic skyrmions. For asymmetric heavy-metal/ ferromagnet/heavy-metal heterostructures, the inversion symmetry is broken by using different layers above and below the ferromagnetic layer, giving rise to an interfacial DMI.

The Hamiltonian describing the DMI between two atomic spins $\mathbf{S}_{i}$ and $\mathbf{S}_{j}$ can be expressed as $\hat{\mathcal{H}}_{\mathrm{DM}}=-\mathbf{D}_{\mathrm{DM}} \cdot\left(\mathbf{S}_{i} \times\right.$ $\mathbf{S}_{j}$ ), where $\mathbf{D}_{\mathrm{DM}}$ is the Dzyaloshinskii-Moriya vector. The direction of the DM vector is defined by the type of DMI in the material. For interfacial DMI, the orientation of the DM vector leads to the stabilization of Néel-type magnetic skyrmions

\footnotetext{
*Corresponding author: susmita.saha@psi.ch (Experiments).

${ }^{\dagger}$ Corresponding author: mateusz.zelent@gmail.com (Simulations).
}

[5], whereas the lack of structural inversion symmetry in bulk materials [6,7] (e.g., B20 materials) leads to the stabilization of Bloch-type magnetic skyrmions [8]. In addition, skyrmions can also be stabilized via the dipolar interaction in systems with small or no DMI [9-11]. The magnetic domain structure stabilized in magnetic layers and multilayer stacks depends on the properties of the magnetic film and magnetic history of the sample, with the final spin configuration of the material determined by the ratio of the uniaxial anisotropy $K_{\mathrm{u}}$ to the demagnetization energy, the thickness of magnetic layer $d$, the strength of the DMI, and the number of layers present in the superlattice. By changing the orientation of the external magnetic field, one can obtain different domain structures during magnetization reversal, such as stripe, labyrinth, nonchiral bubble domains and, in some cases, skyrmions $[12,13]$.

Additionally, the magnetic properties of a film can be influenced via patterning. For example, magnonic crystals $[14,15]$, which are spatially modulated magnetic thin films, form well-defined spin wave band structures [16] due to their periodicity. One common example of magnonic crystals is a ferromagnetic antidot lattice [17-19] consisting of periodically arranged holes in a ferromagnetic thin film. Antidot lattices have been proposed as a medium for data storage devices, where the information bit would be trapped between two consecutive antidots [20,21]. In addition, antidot lattices patterned in magnetic multilayers with high PMA have been 


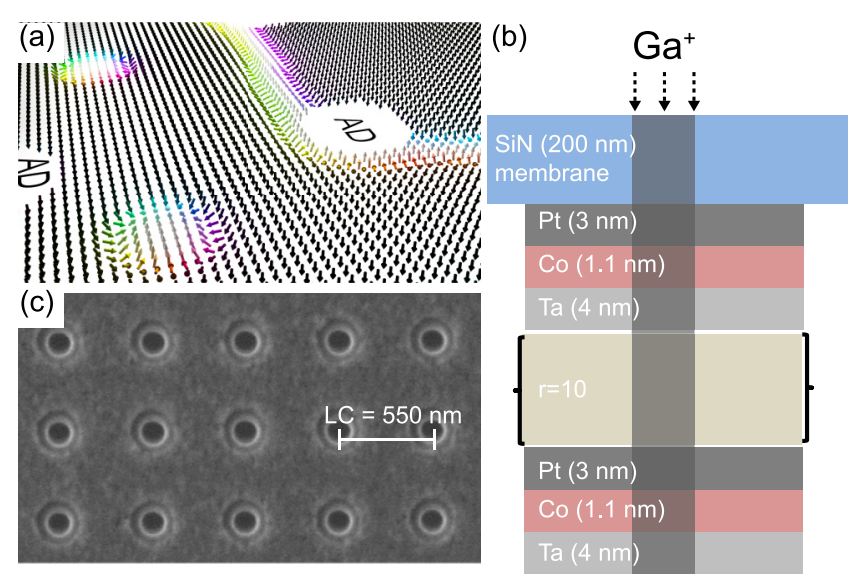

FIG. 1. (a) Visualization of the 3D spin orientation for skyrmions inside the antidot lattice in an applied magnetic field of $-35 \mathrm{mT}$ taken from micromagnetic simulations. (b) Schematic of the multilayer stack with an antidot fabricated using focused $\mathrm{Ga}$ ion beam milling (FIB). (c) Scanning electron microscope image of the antidot lattice with antidot diameter $d=250 \mathrm{~nm}$ and lattice constant $\mathrm{LC}=$ $550 \mathrm{~nm}$ patterned in the $[\mathrm{Pt} / \mathrm{Co} / \mathrm{Ta}]_{12}$ film.

considered as a candidates for perpendicular percolated media [22], which constitutes an alternative approach to conventional bit patterned media. Antidots can also be employed to modify the intrinsic anisotropy of the system [23]. With regard to skyrmions, it has been shown that the edge of a magnetic element as well as defects [24] can repel the skyrmions. In an antidot lattice, the skyrmions are repelled from the edges of the holes, which helps to confine the skyrmions to the saddlepoint region [25]. It has also been shown that pinning sites can accelerate skyrmion motion [24]. An antidot lattice can also give rise to a periodic potential similar to that of an array of pinning sites and therefore could also be used to control current driven skyrmion motion. However, a complete understanding of the influence of an antidot lattice on skyrmion formation during magnetization reversal is still required.

Here, we elucidate the skyrmion formation process in an antidot lattice using both experiment and micromagnetic simulation. In particular, the introduction of holes in an antidot lattice leads to inhomogeneity in the effective field, which has an impact on the formation of the skyrmions. Experimentally, we have fabricated antidot lattices with various lattice constants in a multilayer stack exhibiting both PMA and interfacial DMI (see Fig. 1). Imaging the skyrmions using scanning transmission x-ray microscopy (STXM), we observe that the skyrmions are created from a labyrinth domain pattern on application of a magnetic field. Additionally, we have shown that we can increase or decrease the probability of skyrmion formation by simply varying the lattice constant of the antidot array. From micromagnetic simulations, we have identified the role of the inhomogeneous effective field in the formation of skyrmions in an antidot lattice.

\section{SAMPLE FABRICATION}

Multilayer films of $[\mathrm{Pt}(3 \mathrm{~nm}) / \mathrm{Co}(1.1 \mathrm{~nm}) / \mathrm{Ta}(4 \mathrm{~nm})]_{12}$ were deposited on 200-nm-thick x-ray transparent $\mathrm{Si}_{3} \mathrm{~N}_{4}$ membranes supported by $200-\mu \mathrm{m}$-thick Si frames. The films were deposited using DC magnetron sputtering (AJA International sputter system) with a confocal sputter up geometry at a base pressure of $2 \times 10^{-8}$ Torr. The deposition of all of the layers was performed at a 3 mTorr Ar partial pressure. The antidot lattice was then patterned with focused ion beam milling (FIB) using a focused $30 \mathrm{kV} \mathrm{Ga}^{+}$beam [Fig. 1(b)] with a beam current of $10 \mathrm{pA}$ and a Gaussian spot size of $13 \mathrm{~nm}$. Not only does the incident $\mathrm{Ga}^{+}$ion beam modify the magnetic properties of the film, but also the scattered $\mathrm{Ga}^{+}$ ions, resulting in a lateral distribution of the deposited dose. To reduce the effect of the scattered ions, the ion milling was carried out from the back of the membrane. In this case, the $\mathrm{Si}_{3} \mathrm{~N}_{4}$ membrane absorbs most of the scattered $\mathrm{Ga}^{+}$ions. Two different types of antidot lattices were fabricated with a hole (antidot) diameter $d$ of $250 \mathrm{~nm}$ and lattice constant (LC) of 550 and $1000 \mathrm{~nm}$. A scanning electron micrograph of one of the patterned antidot lattices is shown in Fig. 1(c).

\section{MAGNETIC CHARACTERIZATION}

The magnetic properties of the sputtered films were determined using various techniques. From the out-of-plane hysteresis loop, measured using a superconducting quantum interference device vibrating sample magnetometer (SQUIDVSM; Quantum Design MPMS3) and shown in Fig. 2(a), the saturation magnetization, $M_{\mathrm{s}}$, was found to be $\approx 0.771 \times$ $10^{6} \mathrm{~A} / \mathrm{m}$ and the saturation magnetic field $\approx 40 \mathrm{mT}$. The interfacial DMI of the system was determined by measuring the nonreciprocal spin wave dispersion using Brillouin light scattering (BLS). The presence of the interfacial DMI induces a frequency difference, $\Delta f$, between oppositely propagating Damon-Eshbach modes. This exhibits a linear dependence on the spin wave vector $k$ and is proportional to the effective DMI constant $\mathbf{D}_{\mathrm{DM}}$, according to the following relation:

$$
\Delta f=\frac{2 \gamma \mathbf{D}_{\mathrm{DM}}}{\pi M_{\mathrm{s}}} k,
$$

where $\gamma$ is the gyromagnetic ratio. The spin wave nonreciprocity, $\Delta f$, was determined by measuring the frequency difference between the Stokes (negative frequencies) and anti-Stokes (positive frequencies) peaks in the BLS spectra, which correspond to spin waves propagating in the opposite directions [26].

BLS measurements were carried out by focusing a monochromatic laser $(\lambda=532 \mathrm{~nm})$ on the sample surface and analyzing the backscattered light by a Sandercock-type $(3+3)$ pass tandem Fabry-Perot interferometer. An in-plane magnetic field, sufficiently large to saturate the magnetization in the film plane, was applied along the direction perpendicular to the wave vector of the spin waves. Due to the photonmagnon conservation law of momentum in the scattering process, the magnitude of the wave vector is linked to the incidence angle of light $\theta$, by the relation $k=4 \pi \sin \theta / \lambda$. For our measurements, $\Delta f$ is measured as a function of $k$ for an applied bias magnetic field, $B$, of 0.9 and -0.9 T. $k$ was varied from 0 to $2 \times 10^{7} \mathrm{rad} / \mathrm{m}$ and a linear behavior is observed [Fig. 2(b)]. Note that, upon reversing the direction of the applied magnetic field, $\Delta f$ changes sign due to the reversal of the spin wave propagation direction. The effective DMI constant was then determined with a linear fit [continuous 
(a)

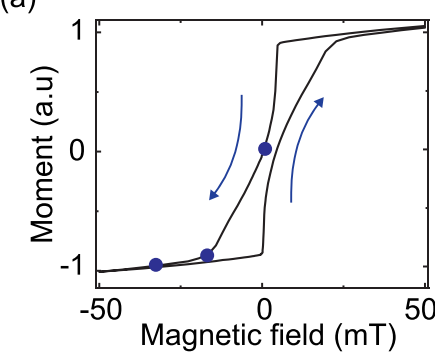

(b)
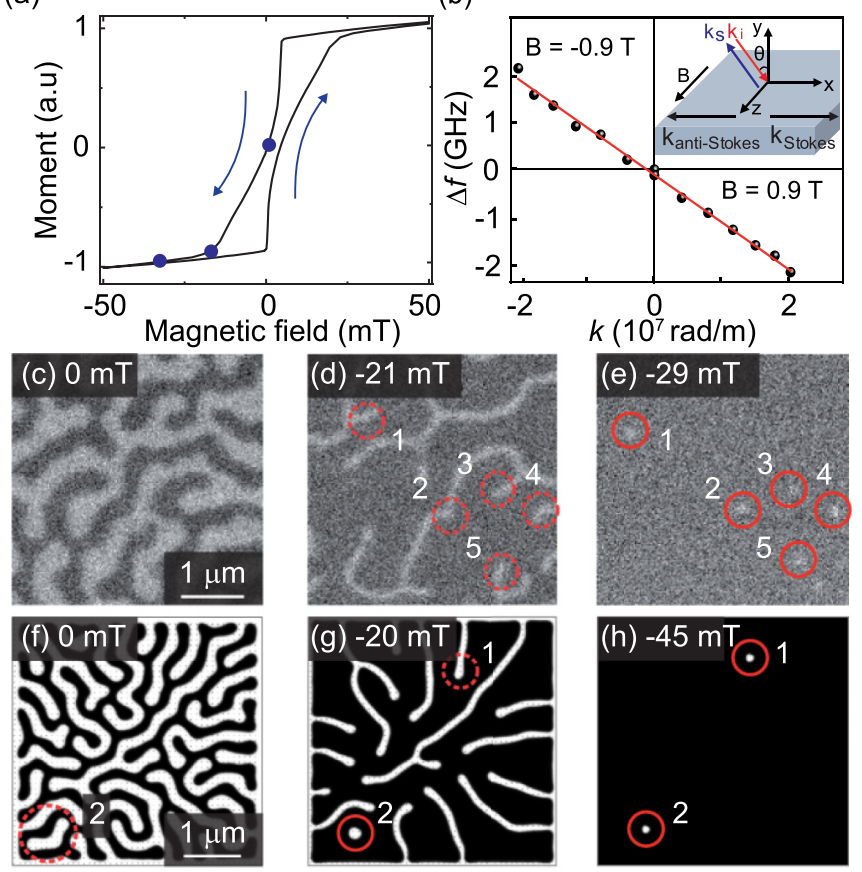

FIG. 2. (a) Out-of-plane hysteresis loop of the $[\mathrm{Pt} / \mathrm{Co} / \mathrm{Ta}]_{12}$ film. (b) The frequency asymmetry $(\Delta f)$ extracted from the BLS measurement of the $[\mathrm{Pt} / \mathrm{Co} / \mathrm{Ta}]_{12}$ film for an in-plane applied bias magnetic field of 0.9 and -0.9 T. Black dots are the experimental results, while the red solid line is the linear fit to the experimental data. $\Delta f$ is the difference between the frequency of the Stokes and the anti-Stokes peaks present in the BLS spectra. A schematic of the experimental configuration is shown in the inset. (c)-(e) XMCD-STXM images of magnetic states in the multilayer film at three different magnetic fields indicated with blue points on the hysteresis loop in (a). (f)-(h) Simulated images of similar magnetic configurations in the multilayer film. In all images, the dark and bright contrast corresponds to domains with magnetization pointing up and down, respectively. The skyrmions are indicated by solid red circles and the domain ends from which the skyrmions are formed are indicated by dashed red circles.

red solid line in Fig. 2(b)] to the experimental data based on Eq. (1). The value of DMI, $\mathbf{D}_{\mathrm{DM}}=-0.78 \pm 0.02 \mathrm{~mJ} / \mathrm{m}^{2}$, was determined using the gyromagnetic ratio $\gamma=194 \mathrm{GHz} / \mathrm{T}$ and the measured value of $M_{\mathrm{s}}$. The value of the DMI is sufficient to support the formation of Néel skyrmions. Moreover, the negative value of $\mathbf{D}_{\mathrm{DM}}$ indicates that left-handed chirality is favored in this material system.

The magnetic configuration of the antidot lattices was imaged using STXM at the PolLux endstation of the Swiss Light Source [27]. Circularly polarized photons are tuned to the $L_{3}$ absorption edge of Co $(778 \mathrm{eV})$ and are focused onto a 25-nm spot on the sample using a diffractive Fresnel zone plate with an outermost zone width of $\approx 25 \mathrm{~nm}$. Magnetic contrast was obtained through the x-ray magnetic circular dichroism (XMCD) effect and by illuminating the sample at normal incidence with respect to the surface. A selection of XMCD images of the magnetic states in a continuous $[\mathrm{Pt} / \mathrm{Co} / \mathrm{Ta}]_{12}$ multilayer film acquired under different applied out-of-plane magnetic fields are shown in Figs. 2(c)-2(e). Prior to the acquisition of the images, the following field initialization protocol was applied; the sample was saturated with a $100 \mathrm{mT}$ out-of-plane magnetic field, which was then reduced to $6 \mathrm{mT}$. After the field initialization, a demagnetized sample with labyrinth domains was observed. Then the magnetic field was reduced to zero and a reversed field was applied in increasing steps. As the magnetic field is increased up to the reverse saturation field, the width of the labyrinth domains with magnetization pointing antiparallel to the applied field is reduced from a value of $\approx 200 \pm 35 \mathrm{~nm}$ at $-6 \mathrm{mT}$ to $\approx 150 \pm 35 \mathrm{~nm}$ upon their collapse into several isolated skyrmions at $-23 \mathrm{mT}$. Close inspection reveals that the skyrmions form at the end of the domains. These skyrmions eventually annihilate at an applied magnetic field of $\approx-38 \mathrm{mT}$.

\section{MICROMAGNETIC SIMULATIONS OF SKYRMION FORMATION}

The skyrmion formation process in a thin film and antidot lattices are simulated using MUMAX3 [28-30]. For the simulation, we have used periodic boundary conditions along the out-of-plane axis with six repetitions in both the negative and positive $z$ directions [31]. The simulations were performed for a finite $4.4 \mu \mathrm{m} \times 4.4 \mu \mathrm{m}$ film using a uniformly discretized grid with a cell size of $2.0 \mathrm{~nm} \times 2.0 \mathrm{~nm} \times 1.1 \mathrm{~nm}$, where the thickness of the cell of $1.1 \mathrm{~nm}$ corresponds to the thickness of the magnetic layer in the real sample. For the simulations, we employed the experimentally measured values of $M_{\mathrm{s}}$ and DMI reported in Sec. III. Furthermore, an exchange constant of $A=1 \times 10^{-11} \mathrm{~J} / \mathrm{m}$ and a PMA of $K_{\mathrm{u}}=3.45 \times 10^{5} \mathrm{~J} / \mathrm{m}^{3}$, taken from the literature [32], were used in the simulations. In order to mimic the polycrystalline nature of the sample, a Voronoi tessellation was added in the micromagnetic simulations where, to each grain, a slightly different perpendicular magnetic anisotropy was assigned, drawn randomly from a normal distribution centered about a mean value with a standard deviation of $10 \%$. The variation in the anisotropy is required to reproduce the experimental observations with the simulations, with high anisotropy grains acting as nucleation sites that facilitate sykrmion formation [33].

For continuous thin films, the ratio of the width of domains with magnetization pointing down (in black) to the width of domains with magnetization pointing up (in white) is controlled by the Zeeman energy, the size and morphology of the black and white domains are controlled by the anisotropy and domain wall energy, and the chirality is determined by the DMI. The simulations of the continuous thin film [Figs. 2(f) and $2(\mathrm{~g})]$ exhibit a good qualitative agreement with the experimental images shown in Figs. 2(c)-2(e). In the simulations, a labyrinth domain pattern is formed at zero magnetic field [Fig. 2(f)]. As the magnetic field is increased, the labyrinth domains collapse into individual skyrmions [Figs. 2(d) and 2(e)]. The skyrmions are formed at the end of the white domains in both the experiment and the simulation. However, in the simulations, the magnetic skyrmions were created at a larger magnetic field than that in the experiment. These observed discrepancies between the micromagnetic simulations and the experimental data can be explained by the fact that the simulations were performed at $0 \mathrm{~K}$, neglecting any thermal fluctuations $[34,35]$. 


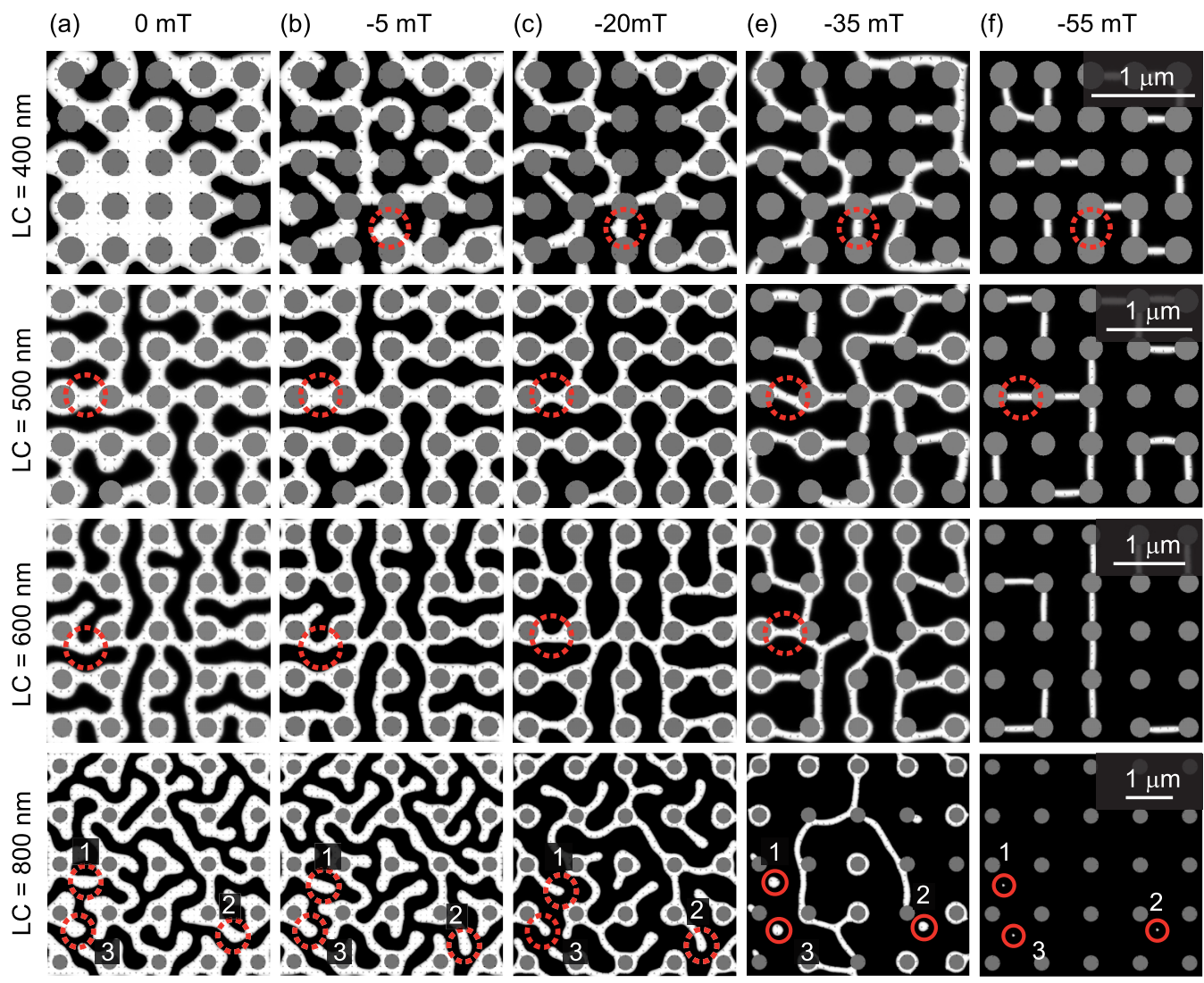

FIG. 3. Simulated images of the evolution of the magnetic configuration in antidot lattices with different lattice constants on increasing the magnetic field. The dark and bright contrast corresponds to domains with magnetization pointing up and down, respectively. Pinned domains are indicated by red dashed circles and the skyrmions are marked with circles with red solid lines.

In order to provide insight into the skyrmion stabilization and their capture in the antidot lattices, micromagnetic simulations were systematically performed for lattice constants of $400,500,600$, and $800 \mathrm{~nm}$. The evolution of the domain patterns on increasing the applied magnetic field is shown in Fig. 3 .

In general, during the magnetization reversal of the antidot lattice, reverse domains (in black) penetrate into the antidot lattice through the channels between the holes. The penetration of the black domains is hindered by the pinning field associated with the antidots, and white domains are pinned around the holes. For the more compact lattices $(\mathrm{LC}=400$, 500 , and $600 \mathrm{~nm}$ ), the labyrinth domain pattern is significantly modified and the details of the magnetization reversal strongly depend on the lattice constant. At $0 \mathrm{mT}$ [Fig. 3(a)] the black domains have penetrated into the antidot lattice for the larger lattice constants, whereas for $\mathrm{LC}=400 \mathrm{~nm}$, most of the lattice is still in a saturated state.

A higher magnetic field is then required for the black domains to penetrate into the $\mathrm{LC}=400 \mathrm{~nm}$ lattice and, due to the lack of space between the antidots, the white domains do not completely surround the holes. For all three lattice constants, the white domains often remain pinned at two neighboring holes on increasing the magnetic field, forming straight narrow domains between them, examples of which are indicated by red dotted circles in Fig. 3 for $\mathrm{LC}=400$, 500 , and $600 \mathrm{~nm}$. With further increase of the magnetic field, these connected white domains shrink to form straight sections between the antidots and eventually vanish at a higher magnetic field. As a result, for these compact lattices, the formation of the skyrmions is hindered due to strong pinning of the domains at the holes in the antidot lattice, shown in the Supplemental Material [36].

For sufficiently large lattice constant, $\mathrm{LC}=800 \mathrm{~nm}$, a partial labyrinth domain pattern is observed at $0 \mathrm{mT}$. In this case, some of the domains are directly connected between two neighboring holes [see, e.g., dashed circles labeled 1 and 2 in Fig. 3(a)] and some form open domain ends [see dashed circle labeled 3 in Fig. 3(a)]. As the applied magnetic field is increased, the connecting domains detach from the holes, forming open domain ends as indicated by the dashed circles labeled 1 and 2 in Fig. 3. With further increase of the applied magnetic field, these open domain ends in the antidot lattice eventually shrink to form magnetic skyrmions indicated by red solid circles in Figs. 3(e) and 3(f). The size of the skyrmions also decreases with the further increase of the magnetic field [compare, e.g., skyrmions in Figs. 3(e) and 3(f), and see Supplemental Material for LC $=800 \mathrm{~nm}$ [37]]. The detailed evolution of the three-dimensional (3D) magnetization configuration on increasing the applied magnetic field for $\mathrm{LC}=400$ and $800 \mathrm{~nm}$ is shown in the Supplemental Material $[38,39]$.

In order to understand the magnetization reversal process and the mechanism behind the skyrmion formation in the antidot lattice, we have extracted the spatial distribution of the effective magnetic field from the simulations for the different 
(a)

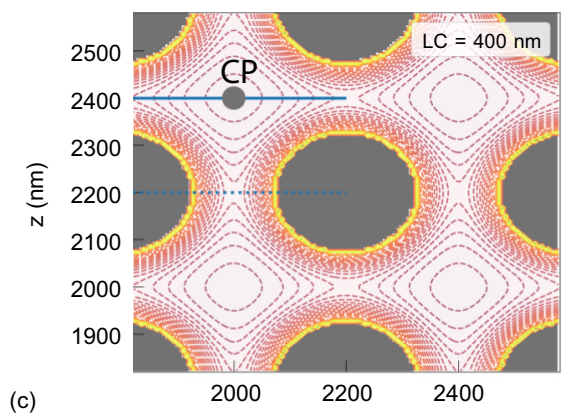

(c)

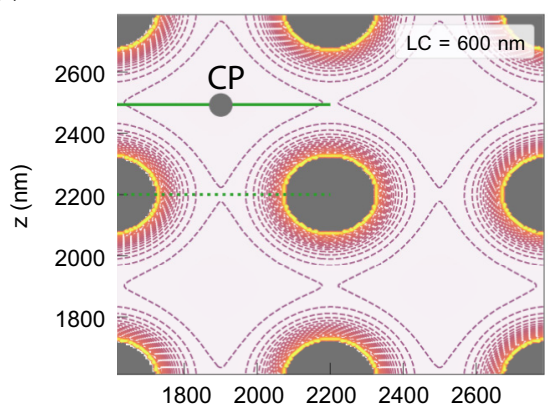

(e)

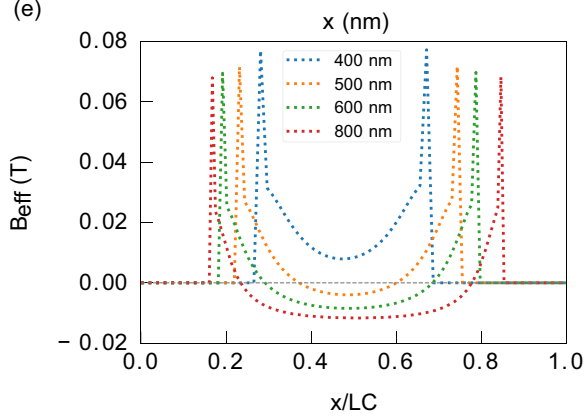

(b)

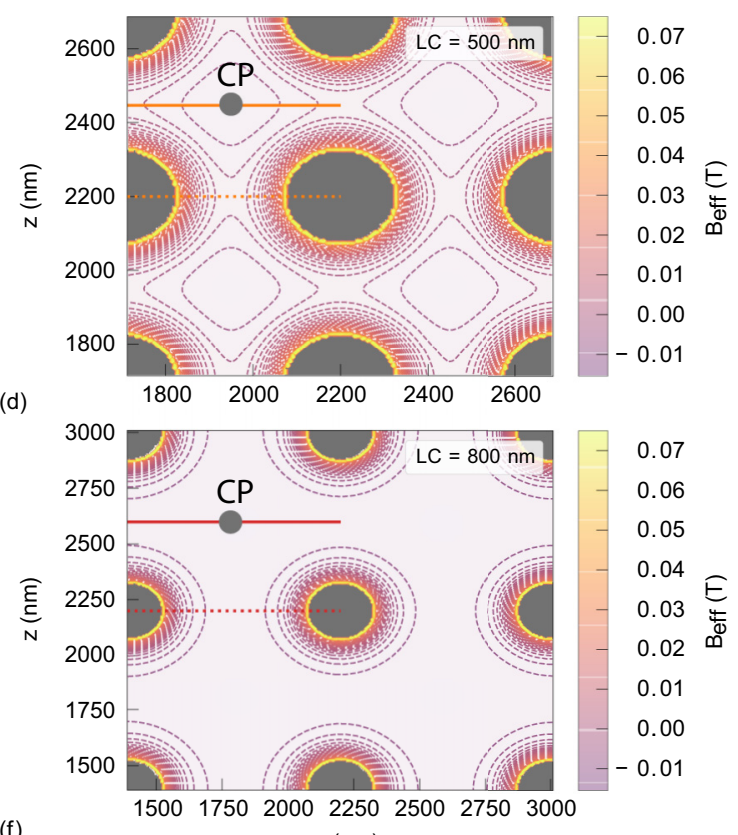

(f)

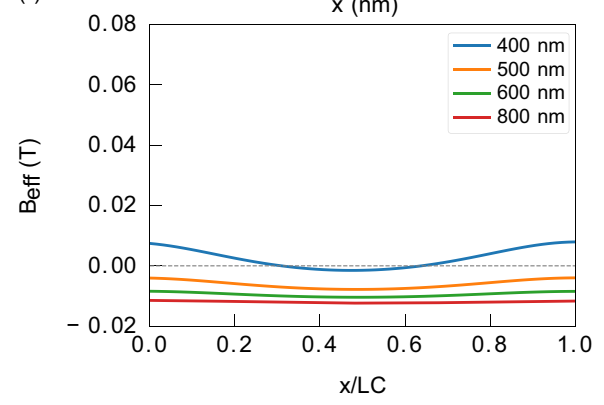

FIG. 4. (a)-(d) Contour plots of the simulated effective field distributions overlaid on the color map for the different lattices at 5 mT. The center point (CP), indicated by the gray dot, is the intersection of the diagonal lines connecting the centers of the antidots. (e), (f) Simulated effective field from different lattices obtained by taking a line scan across the two different regions marked by horizontal dashed and solid lines in Figs. 4(a)-4(d), respectively. Different line colors are used for the different lattices.

lattice constants at a field value of $5 \mathrm{mT}$ so that the system is in a saturated state just before domain formation. The contributions to the effective magnetic field $\left(\boldsymbol{B}_{\text {eff }}\right)$ are from the externally applied field $\left(\boldsymbol{B}_{\text {ext }}\right)$, the magnetostatic field $\left(\boldsymbol{B}_{\text {demag }}\right)$, the Heisenberg exchange field $\left(\boldsymbol{B}_{\text {exch }}\right)$, the DzyaloshinskiiMoriya exchange field $\left(\boldsymbol{B}_{\mathrm{DM}}\right)$, and the magnetocrystalline anisotropy $\left(\boldsymbol{B}_{\text {anis }}\right)$ according to the following equation $[29,40]$ :

$$
\boldsymbol{B}_{\text {eff }}=\boldsymbol{B}_{\text {ext }}+\boldsymbol{B}_{\text {demag }}+\boldsymbol{B}_{\text {exch }}+\boldsymbol{B}_{\mathrm{DM}}+\boldsymbol{B}_{\text {anis }} .
$$

From Figs. 4(a)-4(d), it can be seen that the contour lines of the effective field are very dense around the holes and the line density between the holes decreases with increasing the lattice constant. The profile of effective field along the dashed and solid lines in Figs. 4(a)-4(d) are shown in Figs. 4(e) and 4(f), respectively. From this, it becomes clear that the effective field profile changes, in general decreasing and flattening with the increase of the lattice constant in both the regions. The reversal of the magnetization is facilitated or hindered, depending on whether the out-of-plane component of the effective field is negative or positive, respectively.
Although the variations in the effective field along the profiles is relatively small, it is sufficient to modify the domain patterns. In particular, in Fig. 4(e), it can be seen that the effective field has a high positive value close to the edges of the holes [41,42], which explains the pinning of the white domains around the holes. Additionally, the effective field is a minimum at the intersection of the diagonal lines connecting the antidots, labeled center point $(\mathrm{CP})$, which explains the formation of black domains in the center point region, which is particularly noticeable in the antidot lattices with $\mathrm{LC}=500$ and $600 \mathrm{~nm}$. The larger effective field in the region between neighboring holes supports the pinning of white domains forming a connection between the holes. For an antidot lattice with $\mathrm{LC}=800 \mathrm{~nm}$, the effective field in the channels between the holes [along the solid line in Fig. 4(d)] is almost uniform and, in order to achieve an equilibrium state, the system forms labyrinth domain patterns with open domain ends. As the external magnetic field is increased, the domain width changes to minimize the total energy of the system and, as shown in literature [43], the length of the domains will also decrease to minimize the total energy. During the contraction of the domains, the open domain end is pinned at a local region with 


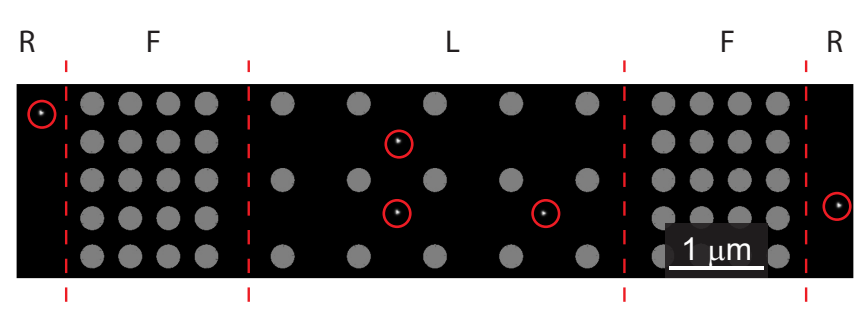

FIG. 5. Snapshot of a micromagnetic simulation showing the magnetic configuration of a composite structure combining two antidot lattices with $\mathrm{LC}=800$ and $400 \mathrm{~nm}$ with an unpatterned film at $H=-50 \mathrm{mT}$. Symbols R, F, and L indicate three different regimes of the structure, where skyrmion formation is at random locations $(\mathrm{R})$, forbidden $(\mathrm{F})$, and localized at the center points in the effective magnetic field (L). For these simulations, the random distribution of the anisotropy of individual grains has not been included.

high anisotropy and, subsequently, a constriction forms that narrows until a skyrmion is formed. This mechanism of formation of skyrmion resembles the necking and detachment of a drop at the edge of a circular capillary tube [44]. We repeated the simulations several times for different distributions in the anisotropy field. The simulated results showed that, while the process of skyrmion formation is always the same, both the number of skyrmions and their position strongly depend on the anisotropy distribution. In general, for an antidot lattice with a uniform anisotropy, the probability of formation of the skyrmions is maximum at the $\mathrm{CP}$, which has the lowest effective field.

In an unpatterned thin film, the skyrmions are spontaneously formed at random positions [45]. However, in our system, the antidot lattice increases the probability of skyrmion formation at a predefined position within the thin film. In order to demonstrate the control of the location of skyrmion formation, we have performed micromagnetic simulations of a composite structure combining a compact $(\mathrm{LC}=400 \mathrm{~nm})$ and a sparser $(\mathrm{LC}=800 \mathrm{~nm})$ antidot lattice together with an unpatterned thin film without a random distribution of anisotropy (Fig. 5). Here, we observe that the skyrmions form at random positions in the film indicated by $\mathrm{R}$ (random), whereas there is a high probability to form the skyrmions at the center points of the antidot lattice with moderate lattice constant indicated by L (localized). In the region with smaller lattice constant, the skyrmion formation is completely forbidden indicated by $\mathrm{F}$ (forbidden). Therefore, it is possible to have skyrmion formation in a selected region of a thin film by fabricating antidot lattice with tailored lattice constants. This concept could be used as a magnetic field induced skyrmion generator for skyrmion-based devices such as skyrmion race track memories, where repeated generation of a skyrmion in a particular location is needed. The skyrmions could then be transferred into other parts of the system, even in the regions where their formation is forbidden, using a spin-polarized current [46].

\section{EXPERIMENTAL OBSERVATIONS}

Following the micromagnetic simulations, we performed experiments on two samples patterned into antidot lattices with different lattice constants, $\mathrm{LC}=550$ and $1000 \mathrm{~nm}$. The XMCD-STXM images of the magnetic configurations in the antidot lattices are shown in Figs. 6(a)-6(h). As for the experiments on the continuous $[\mathrm{Pt} / \mathrm{Co} / \mathrm{Ta}]_{12}$ films, we first performed a field initialization protocol, decreasing the field from $100 \mathrm{mT}$ to zero and then increasing the magnetic field in reverse direction. For the antidot lattice with $\mathrm{LC}=550 \mathrm{~nm}$, the labyrinth domains form connections between the antidots on increasing the magnetic field as indicated by red dashed ellipses in Figs. 6(b)-6(d), which prevents the formation of skyrmions. In contrast, for $\mathrm{LC}=1000 \mathrm{~nm}$, initially the labyrinth domains directly connect two neighboring antidots as indicated, for example, by red dashed circles labeled 1 and 2 in Figs. 6(e)-6(g). On increasing the magnetic field, the domains disconnect from one of the antidots creating an open domain end as indicated by the red dashed circles
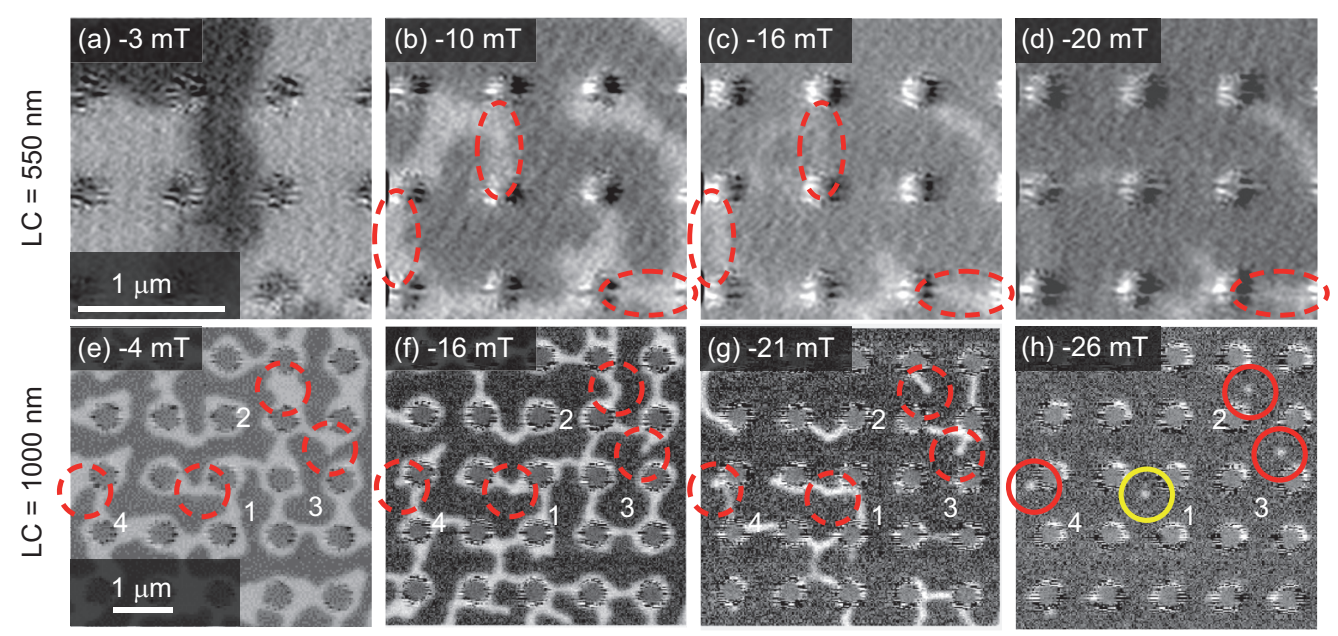

FIG. 6. XMCD-STXM images of the evolution of the magnetic configuration in the antidot lattices with magnetic field for anitdot lattices with (a)-(d) LC $=550 \mathrm{~nm}$ and (e)-(h) LC = $1000 \mathrm{~nm}$. The dark and bright contrast corresponds to magnetic domains with magnetization pointing up and down, respectively. The skyrmions are indicated by solid circles and the domains from which the skyrmions are formed are indicated by red dashed circles and ellipses. 


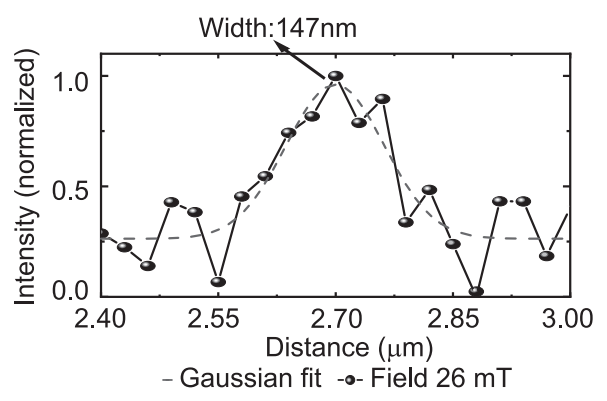

FIG. 7. Line profile of the magnetic contrast across the skyrmion marked with a yellow solid circle in Fig. 6(h). The profile is fitted with a Gaussian curve and has a full width half maximum of $147 \mathrm{~nm}$.

labeled 2, 3, and 4 in Fig. 6(g). The domains with open ends progressively shrink down in width and length until they collapse into isolated magnetic skyrmions indicated by red and yellow solid circles in Fig. 6(h). This experimental observation that skyrmions only form for larger lattice constants is consistent with the results of the micromagnetic simulations. In particular, we note that the mechanism for the skyrmion formation, which occurs at domain ends that only appear for large lattice constants, is observed in both the experiment and simulation.

The domains marked by red dashed circles in Fig. 6 collapse into isolated skyrmions at an applied field of about $-22 \mathrm{mT}$. The diameter of one of the skyrmions [indicated by the yellow circle in Fig. 6(h)] was determined to be $147 \pm$ $35 \mathrm{~nm}$ (see Fig. 7). This is comparable with the simulated skyrmion size, which decreases from 200 to $100 \mathrm{~nm}$ as the field is increased from -35 to $-55 \mathrm{mT}$ although, in the experiment, no significant change in the skyrmion size on increasing the magnetic field could be seen. The skyrmions finally annihilate at a higher applied field and, on repeating the initialization protocol and increasing the field, we observed that the magnetic skyrmions can be repeatedly created in the antidot lattice. With regard to the skyrmion size, DMI leads to stabilized nanometer-sized skyrmions, whereas the larger sized skyrmions are mostly stabilized by the dipolar interaction $[47,48]$. In our case, the size of the observed skyrmions indicates that they are mostly stabilized by the dipolar interaction but the effect of DMI cannot be ignored.

\section{DISCUSSION}

In summary, we have fabricated magnetic antidot lattices with $\mathrm{LC}=550$ and $1000 \mathrm{~nm}$ in a multilayered $[\mathrm{Pt} / \mathrm{Co} / \mathrm{Ta}]_{12}$ multilayer film with perpendicular magnetic anisotropy and DMI, and have imaged the evolution of the magnetic configuration in the presence of an applied magnetic field with scanning transmission x-ray microscopy. Our experimental results correspond to the predictions obtained with micromagnetic simulations. Not only do we observe the formation of Néel skyrmions for the sample with larger lattice constant $(\mathrm{LC}=1000 \mathrm{~nm})$, but also that the size of the skyrmions is comparable with the predictions of the micromagnetic simulations. With both experiment and simulations, we elucidate the mechanism of the Néel skyrmion formation within an antidot lattice. In particular, the skyrmions are created at unconnected domain ends, which are necessary for the formation of the skyrmions in the antidot lattices.

Our micromagnetic simulations confirmed the existence of two distinct regimes, one characterized by small lattice constant $\mathrm{LC} \leqslant 600 \mathrm{~nm}$ that suppresses the formation of skyrmions, while skyrmions are formed for an antidot lattice with LC $\geqslant 800 \mathrm{~nm}$ in the second regime. We showed that the introduction of the holes in a thin magnetic film modifies the spatial distribution of the effective magnetic field, which has a profound influence on the formation of the skyrmions. In particular, the skyrmion formation only occurs at the large lattice constants. In our experimental system, which has random variation of the local anisotropy, the skyrmions form in regions with high anisotropy. However, for an antidot lattice where random variation of the anisotropy is excluded, the skyrmions are formed at the center points of the antidot lattice, where the effective field is a minimum. This property can be exploited to design patterns in magnetic thin films to create skyrmions at predefined locations, while suppressing their formation in other regions.

Recently, it has been reported that, in the presence of a spin-polarized current, the skyrmion trajectory is modified by the defects $[24,46]$. These defects behave as a series of potential barriers for the skyrmions and interact with the skyrmions to modify their trajectory. The array of artificial defects introduced in the form of an antidot lattice can also be used to modify the skyrmion trajectory in the presence of a spin-polarized current. In addition, the potential barriers created by the antidot lattice can be varied by changing the lattice constant of the system.

In conclusion, our demonstration of the role of the effective field in an antidot lattice in the formation of skyrmions is a significant step towards reproducible generation and confinement of stable skyrmions at room temperature. This possibility to control skyrmions is of interest for data storage $[8,49,50]$, reconfigurable skyrmion-based magnonic devices [51], and skyrmion-based race track memory [3].

The data that support this study are available via the Zenodo repository [52].

\section{ACKNOWLEDGMENTS}

We would like to acknowledge Dr. P. Derlet, Staff Scientist at PSI, for his valuable scientific inputs. XMCD-STXM measurements were performed at the PolLux endstation installed at the X07DA beamline of the Swiss Light Source, Paul Scherrer Institute, Switzerland. We thank Dr. E. Müller and A. Weber for sample fabrication. S.S. acknowledges support from an ETH Zurich Post-Doctoral fellowship and Marie Curie actions for People COFUND program (Grant No. FEL11 16-1). M.M. acknowledges funding from the EU FP7 SASPRO Programme (REA Grant Agreement No. 609427, project WEST 1244/02/01) with further cofunding by the Slovak Academy of Sciences. A.K.S. acknowledges funding from the European Community's Seventh Framework Program (FP7/2007-2013) under Grant No. 290605 (COFUND: PSI-FELLOW). M.Z. and M.K. acknowledge funding from the EU Horizon 2020 project MagIC Grant No. 644348, 
Polish Ministry of Science and Higher Education resources for science in 2017-2019 (W28/H2020/2017), National Science Centre of Poland Grant No. 2017/27/N/ST3/00419, and Adam Mickiewicz University Foundation. The simulations were partially performed at the Poznan Supercomputing and Networking Center (Grant No. 398). S.F. acknowledges funding from the EU Horizon 2020 project MagicSky (Grant No. 665095). The PolLux endstation was financed by the German Ministerium für Bildung und Forschung (BMBF) through Contracts No. 05KS4WE1/6 and No. 05KS7WE1. S.T. acknowledges financial support by the European Metrology Programme for Innovation and Research (EMPIR), under Grant Agreement No. 17FUN08 TOPS.
[1] U. K. Rößler, A. N. Bogdanov, and C. Pfleiderer, Spontaneous skyrmion ground states in magnetic metals, Nature (London) 442, 797 (2006).

[2] A. Fert, V. Cros, and J. Sampaio, Skyrmions on the track, Nat. Nanotechnol. 8, 152 (2013).

[3] S. Zhang, A. A. Baker, S. Komineas, and T. Hesjedal, Topological computation based on direct magnetic logic communication, Sci. Rep. 5, 15773 (2015).

[4] O. Boulle, J. Vogel, H. Yang, S. Pizzini, D. de Souza Chaves, A. Locatelli, T. O. Menteş, A. Sala, L. D. Buda-Prejbeanu, O. Klein, M. Belmeguenai, Y. Roussigné, A. Stashkevich, S. M. Chérif, L. Aballe, M. Foerster, M. Chshiev, S. Auffret, I. M. Miron, and G. Gaudin, Room-temperature chiral magnetic skyrmions in ultrathin magnetic nanostructures, Nat. Nanotechnol. 11, 449 (2016).

[5] G. Chen, J. Zhu, A. Quesada, J. Li, A. T. N'Diaye, Y. Huo, T. P. Ma, Y. Chen, H. Y. Kwon, C. Won, Z. Q. Qiu, A. K. Schmid, and Y. Z. Wu, Novel Chiral Magnetic Domain Wall Structure in $\mathrm{Fe} / \mathrm{Ni} / \mathrm{Cu}(001)$ Films, Phys. Rev. Lett. 110, 177204 (2013).

[6] N. Nagaosa and Y. Tokura, Topological properties and dynamics of magnetic skyrmions, Nat. Nanotechnol. 8, 899 (2013).

[7] E. Turgut, H. Paik, K. Nguyen, D. A. Muller, D. G. Schlom, and G. D. Fuchs, Engineering Dzyaloshinskii-Moriya interaction in B20 thin-film chiral magnets, Phys. Rev. Mater. 2, 074404 (2018).

[8] A. Fert, N. Reyren, and V. Cros, Magnetic skyrmions: Advances in physics and potential applications, Nat. Rev. Mater. 2, 17031 (2017).

[9] A. Hrabec, J. Sampaio, M. Belmeguenai, I. Gross, R. Weil, S. M. Chérif, A. Stashkevich, V. Jacques, A. Thiaville, and S. Rohart, Current-induced skyrmion generation and dynamics in symmetric bilayers, Nat. Commun. 8, 15765 (2017).

[10] M. Zelent, J. Tóbik, M. Krawczyk, K. Y. Guslienko, and M. Mruczkiewicz, Bi-stability of magnetic skyrmions in ultrathin multilayer nanodots induced by magnetostatic interaction, Phys. Status Solidi RRL 11, 1700259 (2017).

[11] S. Finizio, S. Wintz, D. Bracher, E. Kirk, A. S. Semisalova, J. Förster, K. Zeissler, T. Weßels, M. Weigand, K. Lenz, A. Kleibert, and J. Raabe, Thick permalloy films for the imaging of spin texture dynamics in perpendicularly magnetized systems, Phys. Rev. B 98, 104415 (2018).

[12] K.-O. Ngand D. Vanderbilt, Stability of periodic domain structures in a two-dimensional dipolar model, Phys. Rev. B 52, 2177 (1995).

[13] J. H. Han, J. Zang, Z. Yang, J.-H. Park, and N. Nagaosa, Skyrmion lattice in a two-dimensional chiral magnet, Phys. Rev. B 82, 094429 (2010).

[14] M. Krawczyk and D. Grundler, Review and prospects of magnonic crystals and devices with reprogrammable band structure, J. Phys.: Condens. Matter 26, 123202 (2014).
[15] P. Malagò, L. Giovannini, R. Zivieri, P. Gruszecki, and M. Krawczyk, Spin-wave dynamics in permalloy/cobalt magnonic crystals in the presence of a nonmagnetic spacer, Phys. Rev. B 92, 064416 (2015).

[16] A. V. Chumak, V. I. Vasyuchka, A. A. Serga, and B. Hillebrands, Magnon Spintronics, Nat. Phys. 11, 453 (2015).

[17] S. Pal, J. W. Klos, K. Das, O. Hellwig, P. Gruszecki, M. Krawczyk, and A. Barman, Optically induced spin wave dynamics in $[\mathrm{Co} / \mathrm{Pd}]_{8}$ antidot lattices with perpendicular magnetic anisotropy, Appl. Phys. Lett. 105, 162408 (2014).

[18] N. Tahir, M. Zelent, R. Gieniusz, M. Krawczyk, A. Maziewski, T. Wojciechowski, J. Ding, and A. O. Adeyeye, Magnetization reversal mechanism in patterned (square to wave-like) Py antidot lattices, J. Phys. D 50, 025004 (2017).

[19] M. Zelent, N. Tahir, R. Gieniusz, J. W. Kłos, T. Wojciechowski, U. Guzowska, A. Maziewski, J. Ding, A. O. Adeyeye, and M. Krawczyk, Geometrical complexity of the antidots unit cell effect on the spin wave excitations spectra, J. Phys. D 50, 185003 (2017).

[20] R. P. Cowburn, A. O. Adeyeye, and J. A. C. Bland, Magnetic domain formation in lithographically defined antidot Permalloy arrays, Appl. Phys. Lett. 70, 2309 (1997).

[21] Z. K. Wang, H. S. Lim, V. L. Zhang, J. L. Goh, S. C. Ng, M. H. Kuok, H. L. Su, and S. L. Tang, Collective spin waves in highdensity two-dimensional arrays of FeCo nanowires, Nano Lett. 6, 1083 (2006).

[22] D. Suess, J. Fidler, K. Porath, T. Schrefl, and D. Weller, Micromagnetic study of pinning behavior in percolated media, J. Appl. Phys. 99, 08CG905 (2006).

[23] A. Y. Toporov, R. M. Langford, and A. K. Petford-Long, Lorentz transmission electron microscopy of focused ion beam patterned magnetic antidot arrays, Appl. Phys. Lett. 77, 3063 (2000).

[24] C. J. O. Reichhardt, D. Ray, and C. J. O. Reichhardt, Quantized transport for a skyrmion moving on a two-dimensional periodic substrate, Phys. Rev. B 91, 104426 (2015).

[25] A. I. Marchenko and V. N. Krivoruchko, Skyrmion-like bubbles and stripes in a thin ferromagnetic film with lattice of antidots, J. Magn. Magn. Mater. 377, 153 (2015).

[26] S. Tacchi, R. E. Troncoso, M. Ahlberg, G. Gubbiotti, M. Madami, J. Åkerman, and P. Landeros, Interfacial Dzyaloshinskii-Moriya Interaction in Pt/CoFeB Films: Effect of the Heavy-Metal Thickness, Phys. Rev. Lett. 118, 147201 (2017).

[27] J. Raabe, G. Tzvetkov, U. Flechsig, M. Böge, A. Jaggi, B. Sarafimov, M. G. C. Vernooij, T. Huthwelker, H. Ade, D. Kilcoyne, T. Tyliszczak, R. H. Fink, and C. Quitmann, PolLux: A new facility for soft x-ray spectromicroscopy at the Swiss Light Source, Rev. Sci. Instrum. 79, 113704 (2008). 
[28] A. Vansteenkiste and B. Van de Wiele, MUMAX: A new highperformance micromagnetic simulation tool, J. Magn. Magn. Mater. 323, 2585 (2011).

[29] A. Vansteenkiste, J. Leliaert, M. Dvornik, M. Helsen, F. GarciaSanchez, and B. Van Waeyenberge, The design and verification of MUMAX3, AIP Adv. 4, 107133 (2014).

[30] J. Leliaert, B. Van de Wiele, A. Vansteenkiste, L. Laurson, G. Durin, L. Dupré, and B. Van Waeyenberge, Current-driven domain wall mobility in polycrystalline Permalloy nanowires: A numerical study, J. Appl. Phys. 115, 233903 (2014).

[31] S. Woo, K. Litzius, B. Krüger, M.-Y. Im, L. Caretta, K. Richter, M. Mann, A. Krone, R. M. Reeve, M. Weigand, P. Agrawal, I. Lemesh, M.-A. Mawass, P. Fischer, M. Kläui, and G. S. D. Beach, Observation of room-temperature magnetic skyrmions and their current-driven dynamics in ultrathin metallic ferromagnets, Nat. Mater. 15, 501 (2016).

[32] R. L. Stoop and P. Tierno, Clogging and jamming of colloidal monolayers driven across disordered landscapes, Commun. Phys. 1, 68 (2018).

[33] X. Yu, M. Mostovoy, Y. Tokunaga, W. Zhang, K. Kimoto, Y. Matsui, Y. Kaneko, N. Nagaosa, and Y. Tokura, Magnetic stripes and skyrmions with helicity reversals, Proc. Natl. Acad. Sci. USA 109, 8856 (2012).

[34] D. Suess, L. Breth, J. Lee, M. Fuger, C. Vogler, F. Bruckner, B. Bergmair, T. Huber, J. Fidler, and T. Schrefl, Calculation of coercivity of magnetic nanostructures at finite temperatures, Phys. Rev. B 84, 224421 (2011).

[35] M. P. Sharrock, Time dependence of switching fields in magnetic recording media (invited), J. Appl. Phys. 76, 6413 (1994).

[36] See Supplemental Material at http://link.aps.org/supplemental/ 10.1103/PhysRevB.100.144435 for the evaluation of twodimensional (2D) magnetization configuration on increasing the applied magnetic field for $\mathrm{LC}=400 \mathrm{~nm}$.

[37] See Supplemental Material at http://link.aps.org/supplemental/ 10.1103/PhysRevB.100.144435 for the evaluation of 2D magnetization configuration on increasing the applied magnetic field for $\mathrm{LC}=800 \mathrm{~nm}$.

[38] See Supplemental Material at http://link.aps.org/supplemental/ 10.1103/PhysRevB.100.144435 for the evaluation of 3D magnetization configuration on increasing the applied magnetic field for $\mathrm{LC}=400 \mathrm{~nm}$.

[39] See Supplemental Material at http://link.aps.org/supplemental/ 10.1103/PhysRevB.100.144435 for the evaluation of 3D magnetization configuration on increasing the applied magnetic field for $\mathrm{LC}=800 \mathrm{~nm}$.
[40] J. Leliaert, M. Dvornik, J. Mulkers, J. De Clercq, M. V. Milošević, and B. Van Waeyenberge, Fast micromagnetic simulations on GPU-Recent advances made with MUMAX3, J. Phys. D 51, 123002 (2018).

[41] J. W. Kłos, D. Kumar, J. Romero-Vivas, H. Fangohr, M. Franchin, M. Krawczyk, and A. Barman, Effect of magnetization pinning on the spectrum of spin waves in magnonic antidot waveguides, Phys. Rev. B 86, 184433 (2012).

[42] V. V. Kruglyak, C. S. Davies, V. S. Tkachenko, O. Y. Gorobets, Y. I. Gorobets, and A. N. Kuchko, Formation of the band spectrum of spin waves in 1D magnonic crystals with different types of interfacial boundary conditions, J. Phys. D 50, 094003 (2017).

[43] R. Wolfe, Applied Solid State Science. Volume 1: Advances in Materials and Device Research (Elsevier Science, Amsterdam, 2013).

[44] D. F. Zhang and H. A. Stone, Drop formation in viscous flows at a vertical capillary tube, Phys. Fluids 9, 2234 (1997).

[45] J. Brandão, D. A. Dugato, R. L. Seeger, J. C. Denardin, T. J. A. Mori, and J. C. Cezar, Observation of magnetic skyrmions in unpatterned symmetric multilayers at room temperature and zero magnetic field, Sci. Rep. 9, 4144 (2019).

[46] D. Suess, C. Vogler, F. Bruckner, P. Heistracher, F. Slanovc, and C. Abert, Spin torque efficiency and analytic error rate estimates of skyrmion racetrack memory, Sci. Rep. 9, 4827 (2019).

[47] A. Bernand-Mantel, L. Camosi, A. Wartelle, N. Rougemaille, M. Darques, and L. Ranno, The skyrmion-bubble transition in a ferromagnetic thin film, SciPost Phys. 4, 027 (2018).

[48] F. Büttner, I. Lemesh, and G. S. Beach, Theory of isolated magnetic skyrmions: From fundamentals to room temperature applications, Sci. Rep. 8, 4464 (2018).

[49] K. Wang, L. Qian, S.-C. Ying, G. Xiao, and X. Wu, Controlled modification of skyrmion information in a three-terminal racetrack memory, Nanoscale 11, 6952 (2019).

[50] Y. Liu, N. Lei, C. Wang, X. Zhang, W. Kang, D. Zhu, Y. Zhou, X. Liu, Y. Zhang, and W. Zhao, Voltage-driven high-speed skyrmion motion in a skyrmion-shift device, Phys. Rev. Appl. 11, 014004 (2019).

[51] M. Mruczkiewicz, P. Gruszecki, M. Zelent, and M. Krawczyk, Collective dynamical skyrmion excitations in a magnonic crystal, Phys. Rev. B 93, 174429 (2016).

[52] S. Saha and M. Zelent, Formation of Néel type skyrmions in an antidot lattice with perpendicular magnetic anisotropy (Version 1.0), https://doi.org/10.5281/zenodo.3478883 (2019). 